\title{
Anesthesia for Temporal Lobe Epilepsy Surgery
}

\author{
Ian A. Herrick and Adrian W. Gelb
}

\begin{abstract}
Anesthetic considerations for temporal lobectomy for refractory epilepsy include ensuring a safe and comfortable perioperative experience for the patient, providing suitable operating conditions for the surgeon, avoiding interference with intraoperative electrocorticographic (ECoG) recordings and facilitating intraoperative functional cortical mapping, if performed. Providing the conditions that simultaneously meet these requirements, using general anesthesia or local anesthesia with sedation, remains a significant challenge for the neuroanesthetist. We review issues pertinent to the choice of anesthetic technique for these procedures.
\end{abstract}

RÉSUMÉ: L'anesthésie pour la chirurgie de l'épilepsie temporale. Assurer que le patient vive une expérience périopératoire sûre et confortable, fournir des conditions opératoires adéquates pour le chirurgien, éviter d'interférer avec les enregistrements électrocorticographiques peropératoires et faciliter la cartographie fonctionnelle corticale peropératoire si cette procédure est utilisée, sont des aspects à considérer quant au choix de l'anesthésie lors de la lobectomie temporale pour une épilepsie réfractaire au traitement. Fournir les conditions qui rencontrent toutes ces exigences, que l'anesthésie générale ou l'anesthésie locale avec sédation soit utilisée, demeure un défi de taille pour le neuroanesthésiste. Nous revoyons les aspects pertinents au choix de la technique d'anesthésie pour ces interventions.

Can. J. Neurol. Sci. 2000; 27: Suppl. 1 - S64-S67

Performing craniotomy with the patient awake continues to represent an important option for the surgical management of patients with refractory epilepsy when intraoperative functional neurologic testing or electrocorticography (ECoG) are needed to define the extent of the corticectomy. The long duration of many neurosurgical procedures, the need to perform intraoperative functional cortical mapping and the unique sedation requirements associated with intraoperative ECoG recording pose substantial challenges for the neuroanesthetist.

Advances in preoperative diagnosis and seizure focus localization and the use of standardized anterior temporal lobe resections have made the use of general anesthesia popular at some epilepsy centres. Provided that intraoperative functional cortical mapping is not required and localization can be achieved with confidence preoperatively, general anesthesia offers the most comfortable option for both the patient and the surgical team.

This article reviews the important considerations associated with the choice of anesthetic technique for temporal lobe epilepsy surgery.

\section{SEDATION FOR EPILEPSY SURGERY UNDER LOCAL ANESTHESIA}

A preoperative visit by the anesthetist represents an important factor contributing to a successful perioperative experience. One of the most important goals of the preoperative visit is to establish a relationship of confidence and trust with the patient. The preoperative visit also provides an opportunity to assess the patient's suitability for a prolonged operation performed under local anesthesia. There are two basic reasons for performing epilepsy surgery under local anesthesia: 1) the need to perform intraoperative functional cortical mapping and 2) the desire to minimize drug-induced interference with intraoperative $\mathrm{ECoG}$ recordings. Circumstances that compromise the ability to achieve these goals, such as young age or behavioral disorders that compromise the ability to cooperate may preclude performing an awake craniotomy. A routine preanesthetic assessment should be conducted and any concomitant medical or psychological concerns addressed. Each patient must be considered a candidate for general anesthesia should intraoperative circumstances warrant (i.e., refractory intraoperative seizures, airway compromise, inadequate regional block, etc.).

From the Department of Anesthesia, University of Western Ontario, London Health Sciences Centre, London, Ontario, Canada.

Reprint requests to: Ian A. Herrick, Department of Anesthesia, London Health Sciences Centre, University Campus, 339 Windermere Rd., London, Ontario, Canada. N6A5A5 
Hence, conditions that may compromise the option of converting intraoperatively to a general anesthetic, such as anticipated difficulty establishing a patent airway, also represent relative contraindications to performing the procedure under regional anesthesia.

Regional anesthetic techniques for blockade of the scalp and dura mater are well established and have been described in detail. ${ }^{1,2}$ A combination of regional blockade of the nerves that innervate the scalp and local anesthetic infiltration of the incision line is typically employed. Although, in experienced centres, local anesthetic scalp blocks provide remarkably consistent anesthesia for craniotomy, few, if any, patients relish the prospect of undergoing a neurosurgical procedure under local anesthesia. Intraoperative sedation is provided to enhance patient comfort by reducing anxiety, promoting relaxation or sedation and supplementing analgesia. Several challenges continue to face the neuroanesthetist despite recent pharmacological advances: 1) to provide the surgeon a suitable operating environment, 2) to give the patient a safe and comfortable perioperative experience and 3) to avoid interference with ECoG recordings.

\section{Choice of sedation drugs and techniques}

During the early intraoperative period the procedure is not particularly uncomfortable if local anesthetic blockade of the scalp and dura mater is adequate. The administration of sedation is intended as an adjunct to the regional anesthetic not as an alternative to compensate for an inadequate block. The objective is to ensure a comfortable patient who is cooperative when intraoperative testing is performed and to minimize druginduced interference when ECoG recording is conducted. Care must be taken to avoid excessive sedation that may result in disinhibition or obtundation with the potential for airway compromise. If sufficient sedation is required during craniotomy to render the patient unconscious and unresponsive, general anesthesia with airway control (tracheal intubation or a laryngeal mask) may be a more prudent choice of technique.

Procedures that require intraoperative functional cortical mapping or ongoing neurological evaluations place few restrictions on the choice of drugs available for intraoperative sedation. In contrast, procedures that involve intraoperative ECoG recording place substantial restrictions on the anesthetic drugs available for sedation since many drugs, including barbiturates, benzodiazepines, etomidate and propofol can suppress epileptiform ECoG activity. ${ }^{3,4,5}$ Traditionally, neurolept analgesia has been the most common sedation technique used during these operations. We typically use droperidol combined with fentanyl. Fentanyl is usually administered as a continuous infusion throughout the procedure. Additional boluses are administered as needed for supplemental analgesia. Fentanyl, sufentanil and alfentanil each possess excellent pharmacologic properties for supplementing intraoperative sedation (i.e., rapid onset, relatively short-acting, potent). Each has been reported to be equally safe and efficacious ${ }^{6}$ and various combinations of droperidol and opioids have been used during neurolept analgesia for epilepsy surgery. ${ }^{7,8}$ In addition to its neuroleptic properties, droperidol is also a potent antiemetic.

Unfortunately, despite the fact that neurolept analgesia produces minimal adverse effects on the ECoG, it is less than ideal as an intraoperative sedative. The distribution and elimination half-lives of fentanyl and droperidol are very different which makes the combination difficult to titrate, particularly in an environment such as the operating room where levels of stimulation are highly variable. Additionally, the neuroleptic properties of droperidol do not consistently promote a sense of sedation and anxiolysis (i.e., a calm and relaxed condition). Instead, neurolept analgesia typically promotes a sense of being disconnected or "locked-in", which some patients find distinctly dysphoric. Despite interest, for the better part of three decades a suitable alternative form of sedation has not been available.

Recently, at some institutions the use of propofol has become popular for sedation during awake epilepsy surgery. ${ }^{9,10}$ Propofol offers excellent properties for prolonged sedation. The rapid onset and short duration of action offer flexibility and ease of titration. As well, propofol's anxiolytic and antiemetic properties, present even at subanesthetic doses, are particularly well suited to craniotomy under local anesthesia.

A variety of sedation techniques involving propofol are in use, largely based on individual preference and experience. We typically administer propofol as a continuous infusion. We have also evaluated the use of propofol patient-controlled sedation (PCS) during epilepsy surgery. This technique allows patients to self-administer doses of propofol throughout the procedure and results in anxiolysis, sedation and satisfaction similar to neurolept sedation. ${ }^{11}$

Administration of an opioid (usually fentanyl or sufentanil) is typically combined with propofol to provide analgesia and supplemental sedation. ${ }^{3}$ Opioids can be administered as intermittent boluses or as continuous infusions. Recently, remifentanil, a highly potent opioid with an extremely short duration of action, has been introduced into clinical practice. Remifentanil, administered as an infusion, is exceptionally titratable and noncumulative. However, its therapeutic margin with respect to ventilatory depression is relatively narrow and an alternate form of analgesia is needed for the transition from the operating room to the postoperative period. The use of remifentanil combined with propofol for sedation during awake craniotomy has been reported ${ }^{12}$ but validation of its efficacy and safety is needed before its widespread use for epilepsy surgery can be advocated.

A significant concern associated with the use of propofol sedation during epilepsy surgery relates to its potential to adversely influence ECoG recordings. ${ }^{13}$ Propofol, in common with other intravenous sedative-hypnotic drugs, induces dosedependent changes in the EEG. Sedative doses of propofol have been reported to depress or obscure epileptiform activity ${ }^{9,14}$ and propofol has been used to terminate status epilepticus. ${ }^{15,16}$ In contrast, activation of nonhabitual ECoG epileptiform activity has also been reported following the administration of propofol. ${ }^{17,18}$ Furthermore, seizure-like movements and other neuroexcitatory phenomena have been well documented in association with the use of propofol..$^{19,20}$ These contrasting issues have raised questions regarding the suitability of propofol for sedation during seizure surgery. We have reported that terminating the administration of propofol sedation at least 15 minutes prior to ECoG recording (usually following dural opening) allows the sedative effects of the drug to dissipate and 
results in ECoG spike frequency, location and propagation comparable to that observed with neurolept analgesia. ${ }^{21}$

\section{GENERAL ANESTHESIA FOR EPILEPSY SURGERY}

Modern diagnostic techniques, including electrocorticography, magnetic resonance imaging and neuropsychological testing, enable the accurate preoperative localization of seizure foci in many patients. Patients with foci localized to noneloquent regions of the temporal lobe are candidates for standardized anterior temporal lobectomy. Using a standardized approach to temporal lobe resection avoids functionally important cortical areas thereby eliminating the need for intraoperative cortical mapping and allows the procedure to be performed under general anesthesia. Some epilepsy centres currently use general anesthesia routinely during these operations. ${ }^{22,23}$

\section{Choice of general anesthetic drugs and techniques}

General anesthetic techniques for epilepsy surgery remain largely empiric, typically institution-specific and generally poorly documented in the literature. A variety of techniques have evolved. The predominant procedure-related consideration regarding the choice of general anesthetic technique during epilepsy surgery relates to the need to minimize anestheticinduced electrocorticographic effects, if $\mathrm{ECoG}$ recording is planned.

In many centres, general anesthesia is maintained throughout the procedure. A combination of nitrous oxide, opioid and low dose volatile anesthetic agent are typically used. ${ }^{23}$ Since each of the volatile anesthetic drugs (i.e., isoflurane, enflurane, halothane, desflurane and sevoflurane) are known to suppress epileptiform activity, even at low to moderate doses, ${ }^{4,5}$ general anesthesia using these drugs carries a risk of obscuring intraoperative ECoG spike activity. Since the level of surgical stimulation is low during ECoG studies, the depth of anesthesia can be reduced to a minimum. The decision to reduce administration of anesthetic drugs to achieve artifact-free recordings is balanced against the risk that some patients may experience intraoperative awareness and recall. In view of the fact that ECoG recording is not associated with discomfort, many patients, appropriately counseled and forewarned, may find this option acceptable.

When the reduction of anesthetic administration is carried to the extreme, anesthesia is suspended and the patient is allowed to emerge from general anesthesia during ECoG recording. This technique is commonly referred to as interrupted general anesthesia. The major drawback to this technique, using traditional anesthetic drugs, is the risk that the patient will become restless, confused or agitated during, or following, emergence. New short-acting anesthetic drugs such as desflurane, sevoflurane, remifentanil and propofol are well suited to such short duration applications and merit formal evaluation. Re-inducing general anesthesia when ECoG recording is complete can also be challenging since patients are typically positioned laterally and may have their heads immobilized in skull pins.

To avoid the need to minimize, or eliminate, the administration of anesthesia during ECoG recording, some interest has been focused on evaluating techniques to augment epileptiform activity during general anesthesia. Methohexital, etomidate and enflurane have been used to evoke spike activity during ECoG recording. ${ }^{4,5}$ Each drug is effective but, controversy continues to surround the issue of whether the elicited spike activity is sufficiently specific to permit accurate localization of the seizure focus. Sevoflurane, ${ }^{24}$ administered in high doses, has also been reported to evoke epileptiform activity among patients with refractory temporal lobe epilepsy, but its use for this purpose during surgery has not been investigated. Low doses of opioids do not appear to possess epileptogenic properties. However, moderately high doses of fentanyl and alfentanil have been reported to trigger spike activity in patients with temporal lobe epilepsy. ${ }^{25-28}$ Spike activity elicited by the administration of alfentanil has been investigated as an adjunct to ECoG recording during epilepsy surgery and is currently a popular technique in some centres. ${ }^{26,28}$

Another option that is used intermittently at our hospital, and likely at other centres where awake craniotomy is performed, is cortical resection under local anesthesia with terminal general anesthesia. In our experience, patients are typically comfortable during craniotomy and the superficial aspects of corticectomy. During temporal lobectomy, discomfort and/or restlessness most commonly accompany mesial temporal resection (i.e., the hippocampus and amygdala). Performing the superficial corticectomy under local anesthesia with sedation minimizes interference with preresection ECoG recordings and allows functional cortical mapping to be performed on patients who are candidates for tailored resection. Conversion to general anesthesia during the terminal phase of the operation contributes to patient comfort and the efficient completion of the procedure. Problems associated with the use of terminal general anesthesia include the need to induce anesthesia and establish a secure airway intraoperatively (similar to interrupted general anesthesia) and potential interference with postresection ECoG recording, if performed. Evidence that postresection ECoG spike activity does not correlate with seizure outcome ${ }^{29}$ has reduced the incentive to obtain these recordings and may enhance interest in the use of terminal general anesthesia during these procedures.

\section{SUMMARY}

The choice of anesthetic technique - local versus general anesthesia, rests predominantly on the choice of surgical technique - standardized versus customized resection. Even among centres with extensive experience performing craniotomy under local anesthesia, the use of general anesthesia typically provides a less stressful environment for the patient, surgeon and anesthetist. Although a variety of general anesthetic techniques can be used during temporal lobe surgery, the adequacy of intraoperative ECoG recording remains controversial and the opportunity to tailor the resection, based on intraoperative ECoG studies, is eliminated. Recent additions to the repertoire of anesthetic drugs and equipment have renewed enthusiasm for developing and evaluating anesthetic techniques that improve patient safety and comfort during these procedures.

\section{REFERENCES}

1. Girvin J. Neurosurgical considerations and general methods for craniotomy under local anesthesia. Internat Anesthesiol Clin 1986;24:89-113.

2. Pinosky M, Fishman R, Reeves $\mathrm{S}$, et al. The effect of bupivacaine 
skull block on the hemodynamic response to craniotomy. Anesth Analg 1996;83:1256-1261.

3. Craen RA, Herrick IA. Seizure surgery: general considerations and specific problems associated with awake craniotomy. Anesthesiol Clin North Amer 1997;15:655-672.

4. Herrick IA. Seizure activity and anesthetic agents and adjuvants. In: Albin MS (ed), Textbook of Neuroanesthesia with Neurological and Neurosciences Perspectives. New York: McGraw-Hill, 1997: 625-642.

5. Modica PA, Tempelhoff R, White PF. Pro- and anticonvulsant effects of anesthetics (Parts I and II). Anesth Analg 1990;70:303315 and 433-444.

6. Gignac E, Manninen P, Gelb A. Comparison of fentanyl, sufentanil and alfentanil during awake craniotomy for epilepsy. Can $\mathbf{J}$ Anaesth 1993;40:421-424.

7. Manninen P, Contreras J. Anesthetic considerations for craniotomy in awake patients. Internat Anesthesiol Clin 1986;24:157-174.

8. Archer D, McKenna J, Morin L, Ravussin P. Conscious-sedation analgesia during craniotomy for intractable epilepsy: a review of 354 consecutive cases. Can J Anaesth 1988;35:338-344.

9. Drummond J, Iragui-Madoz V, Alksne J, Kalkman C. Masking epileptiform activity by propofol during seizure surgery. Anesthesiology 1992;76:652-654.

10. Silbergeld D, Mueller W, Colley P, Ojemann G, Lettich E. Use of propofol (Diprivan) for awake craniotomies: technical note. Surg Neurol 1992;38:271-272.

11. Herrick I, Craen R, Gelb A, et al. Propofol sedation during awake craniotomy for seizures: patient-controlled administration versus neurolept analgesia. Anesth Analg 1997;84:1285-1291.

12. Johnson KB, Egan TD. Remifentanil and propofol combination for awake craniotomy: case report with pharmacokinetic simulations. J Neurosurg Anesth 1998;10:25-29.

13. Sneyd JR. Propofol and epilepsy. Br J Anaesth 1999;82:168-169.

14. Rampil I, Lopez C, Laxer K, Barbaro N. Propofol sedation may disrupt interictal epileptiform activity from a seizure focus. Anesth Analg 1993;77:1071-1073.

15. Pitt-Miller P, Elcock B, Maharaj M. The management of status epilepticus with a continuous propofol infusion. Anesth Analg 1994;78:1193-1194.

16. Yanny H, Christmas D. Propofol infusions for status epilepticus.
Anaesthesia 1988;43:514.

17. Smith M, Smith S, Scott C, Harkness W. Activation of the electrocorticogram by propofol during surgery for epilepsy. Br J Anaesth 1996;76:499-502.

18. Wang B, Bai Q, Jiao X, Wang E, White P. Effect of sedative and hypnotic doses of propofol on the electroencephalogram of patients with and without epileptic activity. Anesth Analg 1997;84:S375(abst).

19. Bevan J. Propofol-related convulsions. Can J Anaesth 1993;40:805809.

20. Orser B. Propofol-induced neuroexcitation and receptor desensitization. Can J Anaesth 1994;41:366-371.

21. Herrick I, Craen R, Gelb A, et al. Propofol sedation during awake craniotomy for seizures: electrocorticographic and epileptogenic effects. Anesth Analg 1997;84:1280-1284.

22. Engel J Jr. Alternate therapy. In: Engel J Jr (ed). Seizures and Epilepsy. Philadelphia: F A Davis, 1989: 443-474.

23. Wass CT, Rajala MM, Hughes JM, et al. Long-term follow-up of patients treated surgically for medically intractable epilepsy: results in 291 patients treated at Mayo Clinic between July 1972 and March 1985. Mayo Clin Proc 1996;71:1105-1113.

24. Watts A, Herrick IA, McLachlan RS, et al. Electrocorticographic effects of sevoflurane and isoflurane in patients with seizure disorders. Anesth Analg 1999;89:1275-1281.

25. Tempelhoff R, Modica PA, Bernardo KL, Edwards I. Fentanylinduced electrocorticographic seizures in patients with complex partial epilepsy. J Neurosurg 1992;77:201-208.

26. Cascino GD, So EL, Sharbrough FW, et al. Alfentanil-induced epileptiform activity in patients with partial epilepsy. J Clin Neurophysiol 1993;10:520-525.

27. Keene DL, Roberts D, Splinter WM, Higgins M, Ventureyra E. Alfentanil mediated activation of epileptiform activity in the electrocorticogram during resection of epileptogenic foci. Can $\mathbf{J}$ Neurol Sci 1997;24:37-39.

28. Manninen PH, Burke SJ, Wennberg R, Lozano AM, El Beheiry H. Intraoperative localization of an epileptogenic focus with alfentanil and fentanyl. Anesth Analg 1999;88:1101-1106.

29. Kanazawa O, Blume WT, Girvin JP. Significance of spikes at temporal lobe electrocorticography. Epilepsia 1996;37:50-55. 